\title{
Modelling biochemical gradients in vitro to control cell compartmentalization in a microengineered 3D model of the intestinal epithelium
}

Gizem Altay, Aina Abad-Lázaro, Emilio J. Gualda, Jordi Folch, Claudia Insa, Sébastien Tosi, Xavier Hernando-Momblona, Eduard Batlle, Pablo Loza-Álvarez, Vanesa Fernández-Majada, and Elena Martinez*

Gizem Altay and Aina Abad-Lázaro should be considered joint first author

Dr. Gizem Altay, Aina Abad-Lázaro, Jordi Folch, Claudia Insa, Dr. Vanesa FernándezMajada, Prof. Elena Martinez

Biomimetic Systems for Cell Engineering Laboratory, Institute for Bioengineering of Catalonia (IBEC), Barcelona Institute of Science and Technology (BIST), Baldiri i Reixac 15-21, Barcelona 08028, Spain

E-mail: emartinez@ibecbarcelona.eu

Dr. Gizem Altay

Institut de l'Audition, Institut Pasteur, INSERM, Université de Paris, Paris 75012, France.

Dr. Emilio J. Gualda, Dr. Matteo Bernardello, Dr. Gustavo Castro, Dr. Jordi Andilla, Dr. Pablo Loza-Álvarez

SLN Research Facility, Institute of Photonic Sciences (ICFO), Mediterranean Technology Park, Av. Carl Friedrich Gauss 3 Castelldefels, Barcelona 08860, Spain

Dr. Sébastien Tosi

Advanced Digital Microscopy Core Facility (ADMCF), Institute for Research in Biomedicine (IRB Barcelona), Barcelona Institute of Science and Technology (BIST), Baldiri i Reixac 10-12, Barcelona, 08028, Spain

Xavier Hernando-Momblona, Prof. Eduard Batlle

Colorectal Cancer Laboratory, Institute for Research in Biomedicine (IRB Barcelona), Barcelona Institute of Science and Technology (BIST), Baldiri i Reixac 10-12, Barcelona, 08028, Spain 
Centro de Investigación Biomédica en Red de Cáncer (CIBERONC), Barcelona, 08028, Spain

Prof. Eduard Batlle

ICREA, Passeig Lluís Companys 23, Barcelona, 08010, Spain

Prof. E. Martinez

Centro de Investigación Biomédica en Red (CIBER), Av. Monforte de Lemos 3-5 Pabellón 11 Planta 0, Madrid, 28029, Spain

Department of Electronics and Biomedical Engineering, University of Barcelona (UB), Martí i Franquès 1, Barcelona, 08028, Spain

Keywords: biomolecular gradients, intestinal stem cell niche, 3D architecture, in-silico modelling, photolithography, light sheet fluorescence microscopy, engineering organoids

Gradients of signaling pathways within the intestinal stem cell (ISC) niche are instrumental for cellular compartmentalization and tissue function, yet how are they formed and sensed by the epithelium is still not fully understood. Here we present a new in vitro model of the small intestine based on primary epithelial cells (i), apically accessible (ii), with native tissue mechanical properties and controlled mesh size (iii), 3D villus-like architecture (iv), and biomolecular gradients of the ISC niche (v). Biochemical gradients are formed through the hydrogel-based scaffolds by free diffusion from a source to a sink chamber. To confirm the establishment of precise spatiotemporally controlled gradients, we employ light-sheet fluorescence microscopy and in-silico modelling. The ISC niche biochemical gradients applied along the villus axis lead to the in vivo-like compartmentalization of the proliferative and differentiated cells, while changing the composition and concentration of the biochemical factors affects the cellular organization along the villus axis. This novel 3D in vitro intestinal model derived from organoids recapitulates both the villus-like architecture and the gradients of ISC biochemical factors, thus opening the possibility to study in vitro the nature of such gradients and the resulting cellular response. 


\section{Introduction}

The small intestine is lined by a monolayer of epithelial cells organized into finger-like protrusions of differentiated cells, called villi, and surrounding proliferative invaginations, called crypts of Lieberkühn. ${ }^{[1,2]}$ The intestinal epithelium undergoes fast and continuous cell renewal driven by the intestinal stem cells (ISCs) residing at the crypt bases. The progeny of the ISCs divide and differentiate while migrating up along the villi, forming distinct stem/proliferative and differentiated cell compartments. ${ }^{[1,3]}$ This compartmentalization is regulated by the interplay of gradients of biochemical factors, microbial metabolites, oxygen and extracellular matrix components formed along the crypt-villus axis. ${ }^{[4-6]}$ The biochemical factors essential for ISC niche maintenance, namely, Wingless-related integration site (Wnt), R-Spondin, and epidermal growth factor (EGF), are only active at the crypt compartments with a decreasing concentration from the crypt base towards the villi..[5] These ISC niche factors arise from multiple sources; on one hand, from the epithelium itself, as Paneth cells found at the crypt bases are well known producers of Wnt3a and EGF factors. ${ }^{[7]}$ On the other hand, subepithelial myofibroblasts (ISEMFs) found below the crypts in the lamina propria have been shown to secrete canonical $\mathrm{Wnt} 2 \mathrm{~b},{ }^{[8,9]} \mathrm{R}-$ Spondins ${ }^{[10]}$ and bone morphogenetic protein (BMP) antagonists (e.g. Noggin, Gremlin 1). ${ }^{[11,12]}$ Although it is known that the gradient of epithelial-autonomous Wnt3a is formed by plasma membrane dilution, ${ }^{[13]}$ it remains to be deciphered which is the nature of all remaining biomolecular gradients coming from either the epithelium or the mesenchyme and how the epithelium integrates those signals.

Traditionally, it has been a challenge to grow intestinal epithelial cells in vitro. Therefore, mouse models have been fundamental in understanding the intestinal epithelial biology and the maintenance of the ISC niche in vivo. Lately, the development of organotypic, three-dimensional (3D) intestinal organoids ${ }^{[14-16]}$ has greatly improved the study of intestinal physiology and pathology in vitro. ${ }^{[17,18]}$ Intestinal organoids are derived from ISCs embedded in a basement membrane matrix (Matrigel), where they expand and selforganize, forming crypt-like and villus-like domains enclosing a lumen. They recapitulate the main cellular components and function of the native tissue. However, their closed geometry is a major limitation when using organoids to better understand the role of biochemical gradients in the tissue homeostasis or restoration. The surrounding 3D matrix prevents the spatiotemporal control of diffusive species present in the cell culture 
medium. In addition, classically cultured organoids are very heterogeneous in size and number of crypts and do not allow an easy access to the lumen. It is, also, acknowledged that the 3D native tissue architecture is fundamental to the formation and maintenance of biomolecular gradients, which subsequently, regulates cell compartmentalization in vivo. ${ }^{[19]}$ In this context, it has been suggested that novel cell culture platforms providing well-controlled microenvironments mimicking the key aspects of the native tissue such as the $3 \mathrm{D}$ architecture and including the spatiotemporal control of diffusive biochemical factors could be instrumental to understand how gradients of such factors regulate gut epithelial organization.

Microfabrication techniques provide a wide set of tools to better mimic the in vivo tissue architecture and function by allowing precise control over the cellular microenvironment. ${ }^{[20-24]}$ Few microengineered systems, reproducing intestinal and colonic architecture, and biomolecular gradients on collagen gels, have proven effective in inducing distinct stem/proliferative and differentiated cell compartmentalization in the monolayers obtained from primary cells. ${ }^{[21,22,25]}$ The colonic arrays obtained in this way were used as a screening platform for microbial metabolites and cytokines. However, the use of a multi-step labor-intensive strategy; natural hydrogels like collagen not being conducive to controlled modifications and being prone to degradation over time, even though reduced by crosslinking; ${ }^{[21,24]}$ and the possible unspecific interactions between the diffusing molecules and the collagen matrix hindering the gradient formation like in the case of rapidly absorbed hydrophobic drugs, ${ }^{[26]}$ pose certain limitations in downstream applications of such culture system. To be of practical use, the fabrication strategy should be easy to implement. Also, synthetic materials, such as poly(ethylene glycol) (PEG), have some advantages over the natural matrices, in allowing better control over biochemical and mechanical properties. In this way, their effect on cellular behavior can be systematically tested facilitating the development of an optimal, well-controlled microenvironment.

Here we employed an in-house-developed, simple and cost-effective photolithographybased technique to fabricate 3D villus-like poly(ethylene glycol) diacrylate (PEGDA)based hydrogels ${ }^{[27]}$ with a controlled mesh size allowing the diffusion of ISC niche biomolecular factors. By developing a custom-made microfluidic device allocating the microstructured hydrogels, we imaged the gradients of a fluorescently labelled model 
protein formed along the vertical axis of the microstructures using a custom-made light sheet fluorescence microscope (LSFM). ${ }^{[28]}$ We then developed an in-silico model matching the experimental data and used the model to predict the gradients of ISC niche factors formed on the microstructures. We adapted these villus-like hydrogels to standard Transwell inserts that permitted access to both sides of the hydrogel, and subsequently, the simple formation of spatiotemporal gradients by using the lower compartment as the source and the upper compartment as the sink. We demonstrated the platform to be suitable for the growth, proliferation and differentiation of intestinal epithelial cells derived from organoids. The presence of the ISC niche biochemical gradients led to the compartmentalization of the proliferative and differentiated cells along the villus axis mirroring those found in vivo. Moreover, changing the biochemical factor composition or the concentration range affected the cellular distribution along the villus axis. The more physiologically representative platform that we present here, combining the $3 \mathrm{D}$ architecture and the gradients of ISC biochemical factors, can be used to systematically test the effect of a wide range of biochemical factors, essential for ISC maintenance and differentiation, on the intestinal cellular response in vitro to better understand the dynamics of the tissue.

\section{Results}

The 3D villus-like microstructured hydrogel scaffolds were fabricated by lithographybased dynamic photopolymerization of PEGDA-AA prepolymer solutions with some modifications to the original protocol. ${ }^{[27]}$ First, the microstructures, and then, the hydrogel base in which they were sustained were formed on flexible porous PET membranes, ready to be assembled into Transwell inserts (Figure $1 \mathrm{~A}$ ). The villus-like microstructures were $350 \pm 44 \mu \mathrm{m}$ in height (Figure $1 \mathrm{~A}$ ) resembling the anatomical dimensions of the small intestine villi. ${ }^{[29-31]}$ The hydrogel bulk elasticity measured for the samples was also consistent with the elastic properties of small intestinal tissue (Figure S1 A). ${ }^{[32]}$

To have a better insight into the protein diffusivity within the network of PEGDA-AA hydrogels, the bulk mesh size $(\xi)$ was estimated using Flory-Rehner equilibrium swelling theory ${ }^{[33,34]}$ as modified by Peppas and Merrill for hydrogels prepared in the presence of water ${ }^{[35,36]}$ (see Supporting Information). The estimated average mesh sizes changed from $11.7 \pm 0.2 \mathrm{~nm}$ to $8.3 \pm 0.2 \mathrm{~nm}$, when the PEGDA concentration was increased from $5 \%$ $\mathrm{w} / \mathrm{v}$ to $10 \% \mathrm{w} / \mathrm{v}$, respectively (Figure $1 \mathrm{~B}$ ), in accordance with the values reported in the 
literature. ${ }^{[37,38]}$ The tuning of the mesh size of the hydrogels by altering the PEGDA concentration is well established in the literature. ${ }^{[39-41]}$ A decrease in the average mesh size hinders the mobility of the polymer chains, limiting the diffusivity of the molecules through the hydrogel. For proper diffusion of the biomolecules, the mesh of the hydrogel network has to be equal to or greater than the diameter of the biomolecules. Taking into account that the hydrodynamic diameters of the main ISC niche biochemical factors (EGF, R-Spondin 1, Noggin, Wnt2b) range from 3.0 to $7.2 \mathrm{~nm}$ (see Table 1), the hydrogels with 5\% - 10\% w/v PEGDA concentration range are suitable for the diffusion of these proteins. On the other hand, there was no significant change in the mesh size for the two different UV exposure times studied, suggesting that maximum macromer conversion has already been achieved at $150 \mathrm{~s}$ of UV exposure.

A

1. Pillars' formation

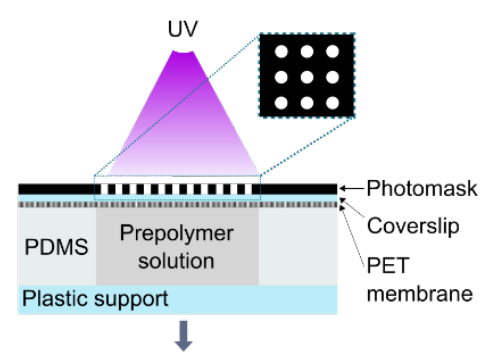

MM

B

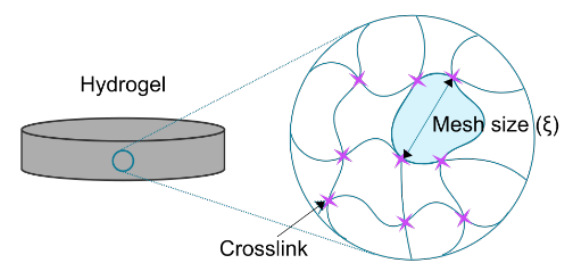

2. Base formation

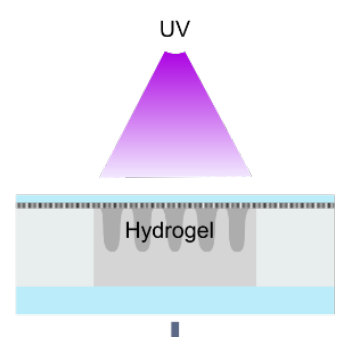

Nonl

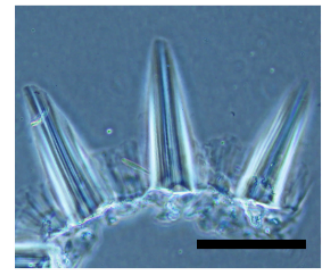

Esite:

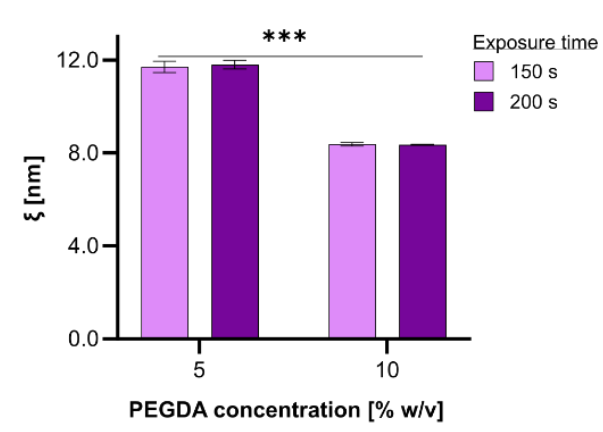

Figure 1. (A) Schematic drawing of the fabrication procedure of villus-like microstructured PEGDA-AA hydrogel scaffolds on porous PET membranes (left and middle). During the first exposure (left), the microstructures were formed using a photomask consisting of an array of circular transparent windows with a diameter and a spacing of $100 \mu \mathrm{m}$ and $150 \mu \mathrm{m}$, respectively. In the second exposure (middle), the photomask was removed and the base with a $6.5 \mathrm{~mm}$ diameter (PDMS pool dimension) was formed. This step was needed to create a homogeneous hydrogel surface. Bright field microscope image of the cross-section of the villus-like microstructured hydrogel 
scaffolds (right). The height of the microstructures was measured to be $350 \pm 44 \mu \mathrm{m}$. $\mathrm{N}=3, \mathrm{n}=4$, pillars $=147$. Mean \pm SD. Scale bar: $400 \mathrm{~mm}$. (B) Schematic representation of the mesh size $(\xi)$ (left). Graph showing estimated mesh size $(\xi)$ for $5 \%$ and $10 \% \mathrm{w} / \mathrm{v}$ PEGDA-AA hydrogels fabricated with $150 \mathrm{~s}$ and $200 \mathrm{~s}$ of UV exposure (n=4) (right). Pvalue: $* * * p<0.001$. Mean \pm SD.

In order to form gradients of ISC niche factors within villus-like microstructured hydrogels and visualize them by microscopy techniques, a custom-made microfluidic device that allowed allocating the PEGDA-AA hydrogel was designed and fabricated. The SU-8 masters were fabricated by photolithography and replica molded in PDMS. The PDMS chip consisted of a microfluidic fluidic channel and a source chamber at the center, allocating the hydrogel sample right above the chamber (Figure 2 A left, Figure S2 A). The hydrogel samples were fabricated on porous membranes and stuck to the microfluidic chip by double-sided pressure-sensitive adhesive (PSA) rings or by using uncured PDMS (Figure 2 A left, Figure S2 A). The protein solutions were delivered through the channel to the source chamber to generate the biomolecular gradients along the villus axis of the microstructured hydrogels. Fluorescently labeled gradients were visualized in-chip by LSFM. $^{[28]}$ LSFM technique was selected as it permits fast imaging (minimal photobleaching) at large working distances with fine axial resolution, a trade-off difficult to achieve by traditional confocal microscopy. ${ }^{[42,43]}$ By decoupling the illumination and detection light paths, LSFM allows a flexible sample access and mounting, which was needed for the microfluidic device (Figure 2 A middle). The chip was designed in a way that the hydrogel scaffold would stick out allowing proper physical access required for imaging (Figure 2 A left, Figure S2 A). To fit the microfluidic chip in the microscope, a custom-made sample holder was designed and fabricated (Figure 2 A right). This had transparent walls permitting the entrance of the light-sheet to the sample and a pedestal with $25^{\circ}$ inclination to have better access to the hydrogel and the source chamber allowing proper imaging (Figure 2 A right).

For the characterization of the biomolecular diffusion dynamics, Texas Red-labelled BSA was selected as the model protein. The diffusion through the microstructured hydrogel scaffolds was imaged by performing a time-lapse acquisition (Figure $2 \mathrm{~B}$ top left). The microfluidic chip allocating the hydrogel was loaded with Texas Red-labelled BSA and an overall 3D volume of $1.33 \times 1.33 \times 2.30 \mathrm{~mm}$ was imaged over 90 minutes. Besides 
Texas Red emission, we also acquired autofluorescence emission from the hydrogels, excited by a $488 \mathrm{~nm}$ laser, obtaining supplementary information about the hydrogel morphology and the membrane interface. The intensity profiles along the perpendicular axis of the hydrogels were taken and converted to concentration values by using a previously established calibration curve within the same setup (Figure $2 \mathrm{~B}$ top right). The concentration profiles showed that a gradient was generated within the hydrogels due to the diffusion of the proteins from the source to the sink. The gradient profiles were stable along the time of acquisition (Figure $2 \mathrm{~B}$ top right), indicating that 3 hours loading time were sufficient to reach a pseudo steady state leading to the formation of stable protein gradients within the hydrogels. On the other hand, the protein accumulation at the PET membrane-hydrogel interface was found to be significant.

In silico modelling of biomolecular diffusion allows to predict the gradient profiles of proteins of interest that would have, otherwise, been tedious to determine experimentally. To that end, an in-silico model of diffusion was developed using finite element method (FEM). In agreement with the experiments performed, BSA was chosen as the model protein to develop the model, and the microstructure geometry and the material accumulation observed at the membrane-hydrogel interface were implemented (Figure 2 B bottom left). The concentration profiles computed along the perpendicular axis spanning the source, the hydrogel and the sink in the geometry designed matching the experimental setup also predicted stable gradients within the timeframe studied, in perfect agreement with the experimental data (Figure 2 B bottom right, Figure S2 B). Moreover, the simulations showed that periodic medium replenishment performed every $24 \mathrm{~h}$ would establish stable gradients, so it was implemented in the cell culture experiments (Figure S3). Then, the in-silico model developed was used to predict the concentration gradients of ISC niche biochemical factors Noggin, R-Spondin 1, and EGF formed along the villus axis of the hydrogels in the cell culture setup, using data from the model proteins BSA, $\mathrm{CA}$, and Insulin, respectively (Figure $2 \mathrm{C}$ top). Simulations revealed that when the source concentrations were doubled (BSA 2.0 and CA 2.0), the concentrations in the entire geometry were also doubled. On the other hand, the percent decrease from base to tip were found to be to be $17.0 \%, 16.9 \%, 14.5 \%, 14.4 \%$, and $7.5 \%$ for BSA, BSA 2.0, CA, CA 2.0 and Insulin, respectively (Figure $2 \mathrm{C}$ bottom), showing that relative change is unaffected by changes in the source concentration. Therefore, our system enables to 
regulate the absolute concentrations that the cells can sense, while keeping the relative drop constant.
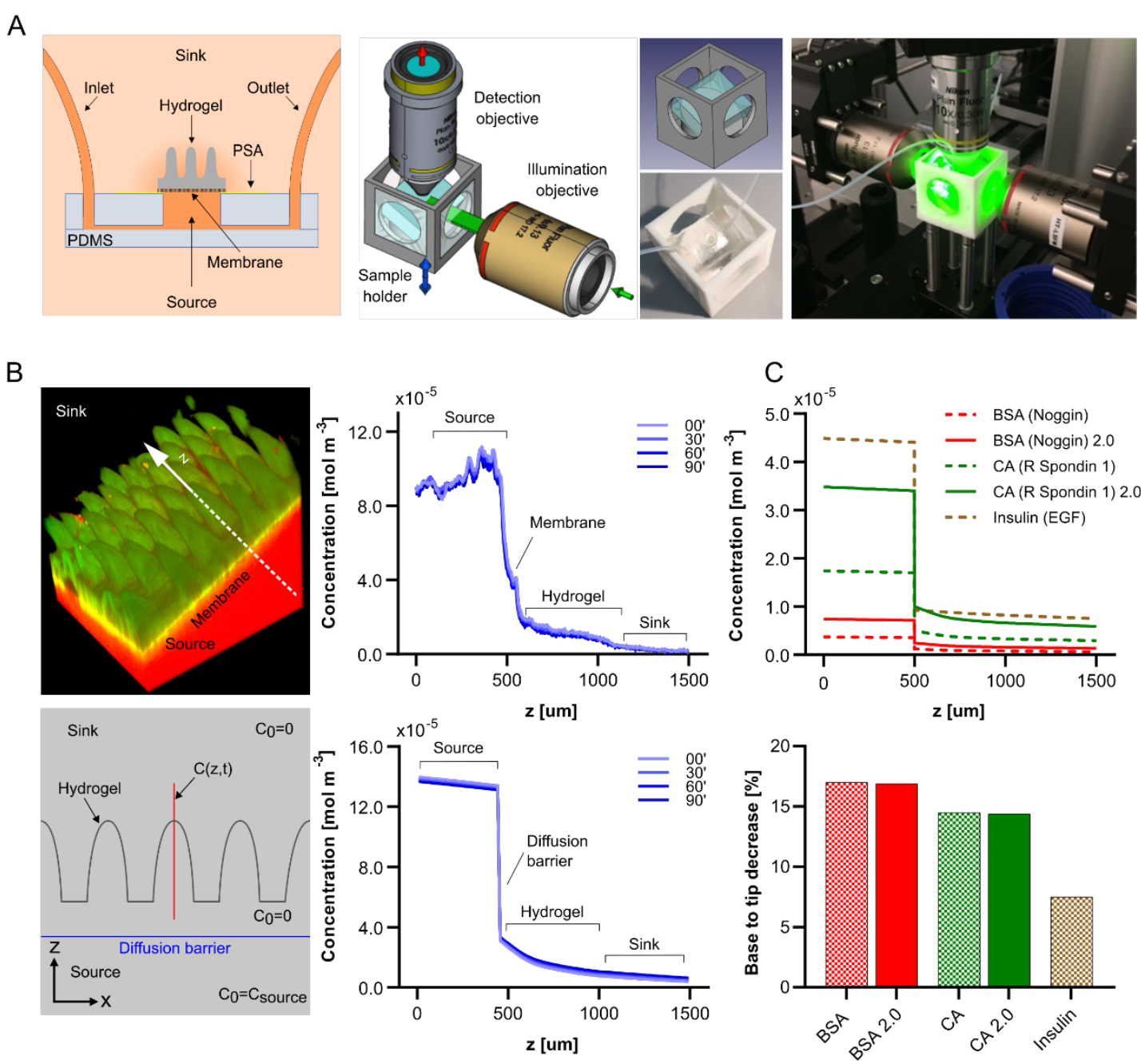

Figure 2. (A) Schematic drawing of the microfluidic chip allocating villus-like microstructured PEGDA-AA scaffold (left). The LSFM setup used, where the detection was performed in an up-right configuration (middle). Schematic of the imaging chamber designed, picture of the microfluidic chip glued to the imaging chamber and the final setup (right). (B) 3D reconstructed fluorescence image of Texas Red-labeled BSA (red) diffusion through villus-like microstructured scaffolds (green) acquired by LSFM spanning the source, the hydrogel and the sink at 3 hours after loading (top left). Graph showing the concentration profiles of Texas Red-labeled BSA taken along the white arrow shown in the left at different times points (top right). The beginning of the timelapse was set as the zero time point. Samples from two independent experiments showed similar results. Scheme showing the microstructure geometry and the initial conditions used in the FEM simulations (bottom left). The initial concentrations were set to zero in 
the hydrogel and the sink, and to the corresponding experimental value in the source. The concentration profiles as a function of time along the red line spanning the source, the hydrogel and the sink were determined. The sink and source sizes are not to scale for visualization purposes. Graph showing the concentration profiles predicted by the FEM simulations of the diffusion of the model protein BSA (bottom right). (C) Graph showing the concentration profiles predicted by the FEM simulations at $24 \mathrm{~h}$ for BSA (Noggin), BSA 2.0 (doubled source concentration), CA (R Spondin 1), CA 2.0 (doubled source concentration) and Insulin (EGF) taken along the red line shown in B bottom left (top). Graph showing the percent decrease in concentrations of the proteins from base to tip of the microstructures (bottom).

After characterizing the gradients of ISC niche factors formed on PEGDA-AA villus-like hydrogels by LSFM and in-silico modelling, we assessed whether we could grow complete organoid-derived epithelium monolayers on the microstructured hydrogels bearing the biochemical gradients. For that, the PEGDA-AA villus-like hydrogels were fabricated, mounted on Transwell inserts and functionalized using collagen type I (Figure 3 A). Then, the proteins were delivered in the Transwell insert asymmetrically to create the spatial gradients. For this first step, we employed the cocktail of factors we use to culture organoids (ENRCV) supplemented to conditioned medium from intestinal subepithelial myofibroblasts (ISEMF_CM) and we corrected the factor concentrations for the protein accumulation taking place in the membrane as predicted by the in-silico simulations. 24 hours after the formation of the gradients, we seeded organoid derived single cells from mouse isolated intestinal crypts. With the aim of optimizing the formation of the epithelial monolayers, we first tested the scaffolds fabricated with microstructures separated by either $100 \mu \mathrm{m}$ or $150 \mu \mathrm{m}$ and found that $150 \mu \mathrm{m}$ spacing provided a sufficient base area for the epithelial monolayer to be formed (Figure S4 A). Also, among the AA content tested in the hydrogel base formulation, it was found that $0.3 \% \mathrm{w} / \mathrm{v}$ AA allowed the formation of monolayers with characteristic epithelial morphology at the base region (Figure S4 B). Finally, we found that the minimum cell seeding density required to obtain a full epithelium on the scaffold was $5 \times 10^{5}$ cells/sample (Figure S4 C).

Having established the optimal conditions for the growth of complete epithelial monolayers, we wondered if the process of monolayer formation was dependent on the presence or absence of ISC niche biomolecular gradients. For this purpose, we seeded 
organoid-derived single cells on top of scaffolds bearing gradients and on scaffolds with no gradients, that is to say, with uniform concentration of the factors. Every 24 hours we renewed the media of both apical and basolateral compartments to maintain the gradients stable (Figure S3). One day after cell seeding, cells were starting to form a monolayer, covering approximately $30 \%$ of the surface under both, asymmetric and uniform conditions (Figure $3 \mathrm{~B}$ ). 48 hours after seeding, the monolayer extended to $70 \%$ of the surface in the asymmetric condition while in uniform condition, the surface was almost fully covered. At 72 hours, the cultures reached nearly 100\% surface coverage in both conditions (Figure $3 \mathrm{~B}$ ). We hypothesize that this faster progression of single cells into full monolayers under uniform condition compared to asymmetric might be due to the greater availability of stem cell niche factors in this condition as factors are not only delivered basolaterally but also apically. Still, it appears that organoid-derived cells were able to form complete monolayers on the surface of the hydrogels regardless of the presence or absence of such gradients. This implies that the type of ISC niche factor gradients formed in our system were not necessary for epithelial cells to be able to form complete epithelial monolayers; provided that the minimum concentration of factors required for cell growth were supplied. Still, it remained to be deciphered whether gradients of ISC factors determine cell compartmentalization in vitro.

A
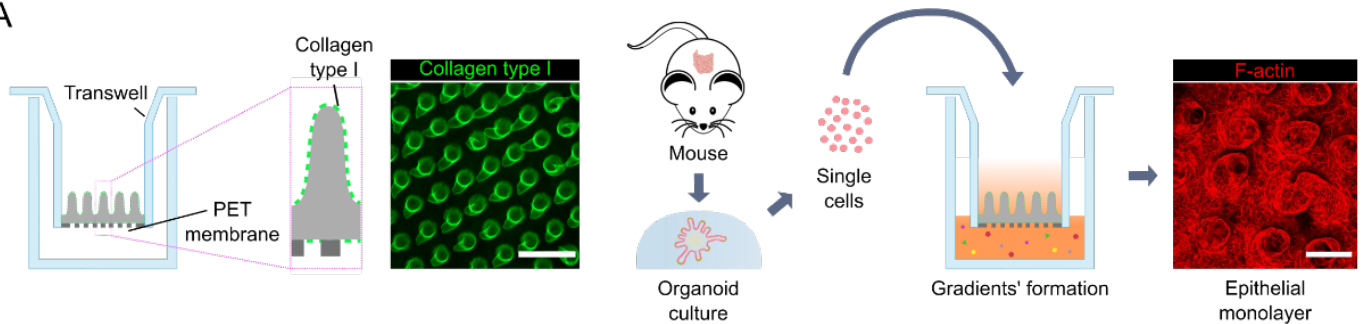

B

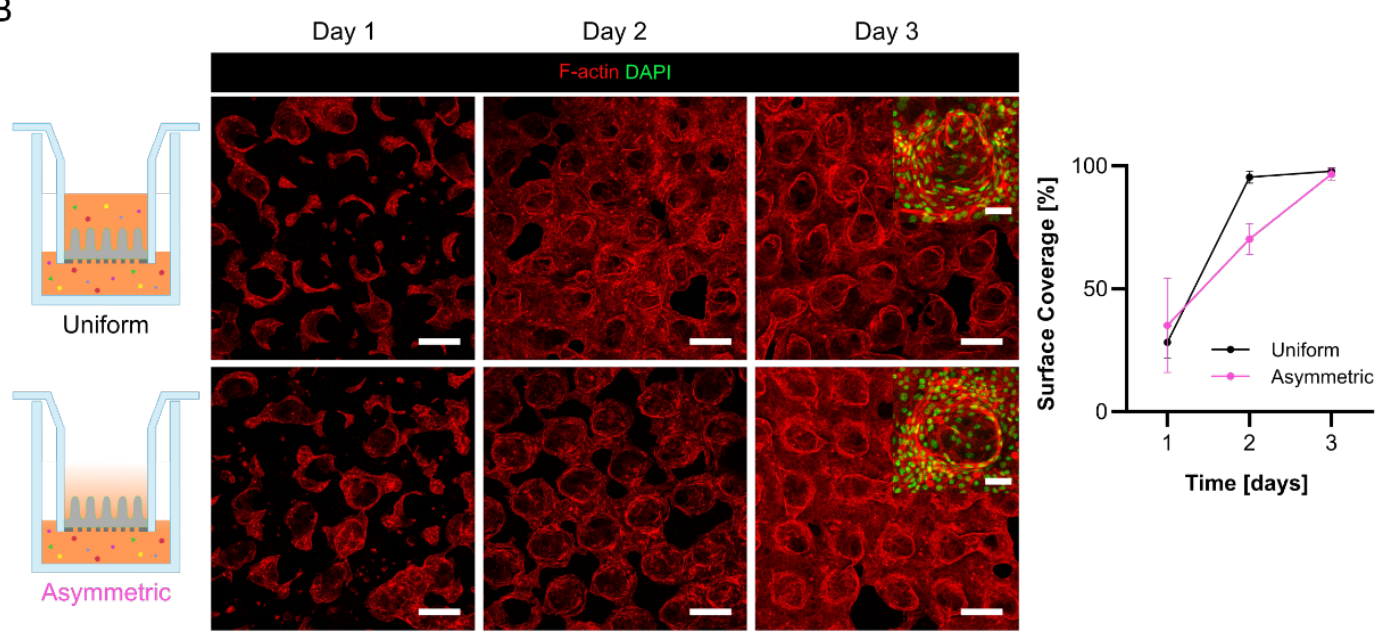


Figure 3. (A) Schematic drawing showing a scaffold mounted onto a Transwell insert and functionalized with collagen type I, an image of a functionalized scaffold, a schematic drawing showing the organoid-derived single cell seeding on collagen functionalized scaffolds with the gradients of ISC niche proteins already generated and an image of the epithelial monolayer formed (from left to right). (B) Schematic drawings of Uniform and Asymmetric conditions. Representative confocal maximum intensity projection images of samples at day 1, 2 and 3 of culture immunostained for F-actin. Scale bars: $200 \mu \mathrm{m}$. Zoom-in insets at day 3 immunostained for F-actin and DAPI. Scale bars: $50 \mu \mathrm{m}$. Graph plotting the percentage of surface covered by epithelial cells with respect to the total surface as a function of the cell culture time in the absence (Uniform) or presence (Asymmetric) of ISC biochemical gradients. $\mathrm{N} \geq 2, \mathrm{n} \geq 2$. Mean $\pm \mathrm{SD}$.

For that aim, we analyzed the location of proliferative $\left(\mathrm{Ki} 67^{+}\right)$cells and differentiated $\left(\mathrm{CK} 20^{+}\right)$cells in the presence or absence of gradients of ISC niche factors when there was a complete epithelium (i.e. at day 3). First, we analyzed the compartmentalization of proliferative $\mathrm{Ki}^{+} 7^{+}$cells along the villus axis. Under gradients of stem cell niche factors, the frequency of proliferative cells positioned at the base was significantly higher (Figure $4 \mathrm{~A}, \mathrm{C}$ left). We observed that almost $50 \%$ of proliferative were located at the base, while only $11 \%$ were at the tip of the pillars. Instead, when we administered the factors uniformly without creating gradients, less than $30 \%$ of the $\mathrm{Ki}^{+}$cells were located at the base, and above $15 \%$ were located at the tip and the difference was not statistically significant (Figure $4 \mathrm{~A}, \mathrm{C}$ left). This suggests that the presence of gradients guides the proliferative cells to localize at the base of the pillars. Moreover, to assess the evolution of this in vivo-like compartmentalization of proliferative cells in culture, we quantified their relative change of frequency at the base and at the tips from day 1 to day 3 . We observed that under asymmetric administration of the factors, the frequency of Ki67 $7^{+}$cells at the base increased significantly with time, while decreasing over the pillars (Figure 4 $\mathrm{C}$ middle), suggesting that the concentrations of factors the cells located at the pillars sensed were not enough for them to maintain the proliferative state. Although a similar tendency was observed under the uniform administration of the factors, the difference was much fainter and not statistically significant (Figure $4 \mathrm{C}$ middle). Then, we wanted to assess how the positioning of the differentiated population in complete epithelium state was impacted by the gradients of stem cell niche factors. For that, we analyzed the mean intensity of CK20, a marker with a graded as opposed to binary expression. ${ }^{[4]}$ In the 
absence of the gradients, the normalized mean intensity of CK20 was similar all along the pillars. In contrast, in the presence of gradients, there was a steep difference of such intensity, being lower at the base and higher at the tip of the pillars, closely resembling the in vivo disposition (Figure $4 \mathrm{C}$ right).

After observing that the gradients of stem cell niche factors formed in our in vitro platform modify the proliferative and differentiated cell positioning, we made use of our 3D in vitro system to modify the gradients applied and to read out the effect on the cellular response. For this purpose, we devised two new conditions: in the first one, we doubled the concentrations of Noggin and R-Spondin 1 (Asymmetric 2.0); and in the second one, we tested a chemically defined medium by plainly substituting ISEMF_CM in Asymmetric 2.0 for $\mathrm{Wnt} 2 \mathrm{~b}$, the most relevant proliferation-promoting molecule coming from the mesenchyme ${ }^{[9,45]}$ (Asymmetric Wnt2b) (Table 1, Table S1). The in-silico model we developed predicted that doubling the source concentration of R-spondin 1 and Noggin would result in doubled concentrations for a given point along the villus axis, while the gradient slopes would stay unchanged (Figure 5 A). Cells receiving doubled amounts of these ISC niche factors account for the increased expansion of the epithelial monolayers we observed at day 1 (Figure 5 B). The surface coverage was $81.4 \%$ and 83.6\% for Asymmetric 2.0 and Asymmetric Wnt2b conditions, respectively, compared to $35.1 \%$ for the Asymmetric condition. At day 3, epithelial cells occupied almost the entire scaffold surface for all the conditions (96.6\% for Asymmetric, 96.5\% for Asymmetric 2.0 and $99.2 \%$ for Asymmetric Wnt2b), therefore constituting a full epithelial monolayer. The faster expansion of the epithelial monolayers under both Asymmetric 2.0 and Asymmetric Wnt2b conditions can be explained by the increased amounts of proliferative cells. At day 1 , the percentage of $\mathrm{Ki}^{+} 7^{+}$proliferative cells was significantly higher in Asymmetric Wnt2b compared to the other two conditions (Figure $5 \mathrm{C}$ top). On the other hand, the frequency of $\mathrm{Ki}^{+} 7^{+}$cells was significantly reduced at day 3 for the same condition, suggesting that the type of $\mathrm{Wnt} 2 \mathrm{~b}$ gradient present in our system leads to an initial fast expansion and a later exhaustion of the proliferative domain, altering the growth rhythm of the epithelial monolayer. 
A

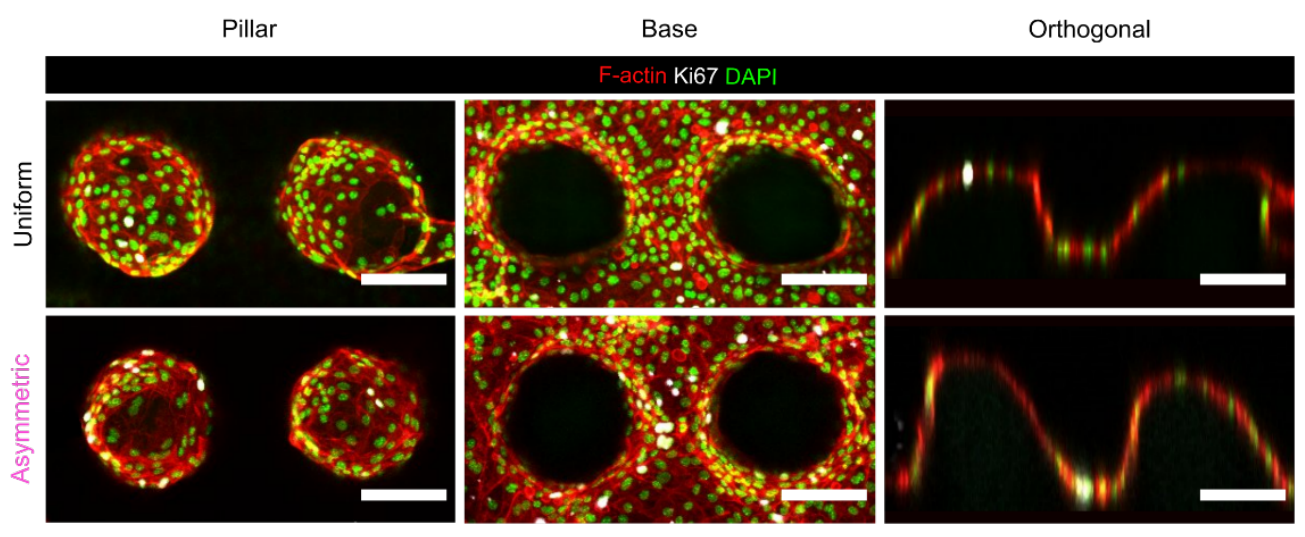

B
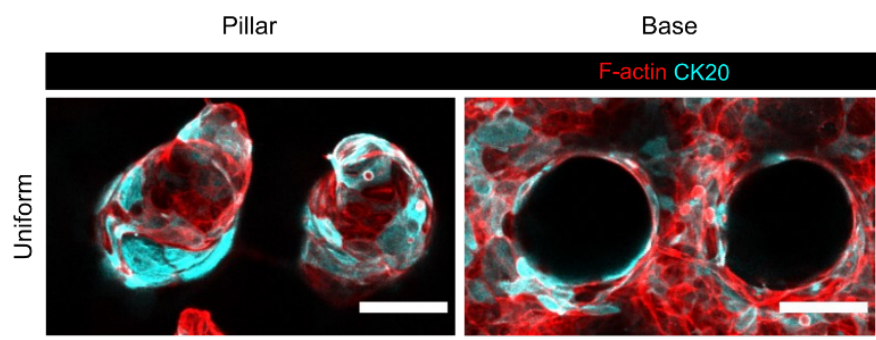

Orthogonal
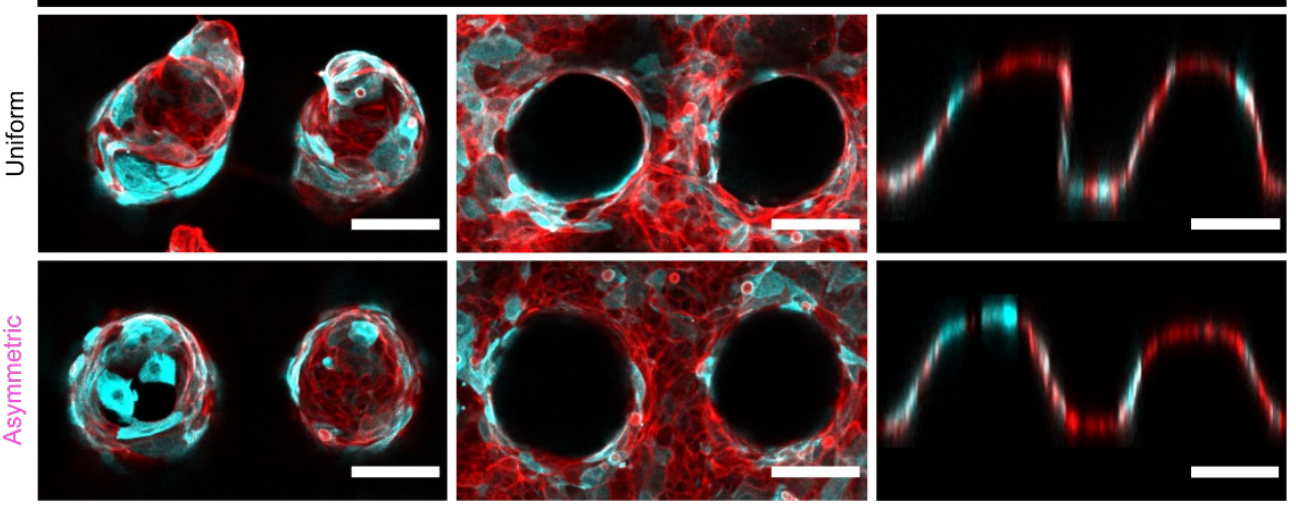

C
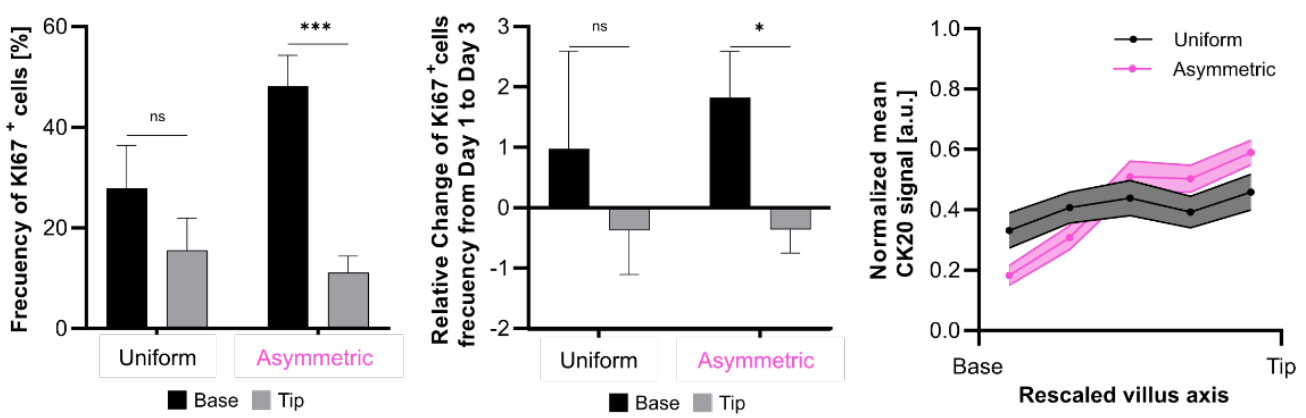

Figure 4. (A) Representative confocal images of Uniform (top) and Asymmetric (bottom) samples on day 3 of culture immunostained for F-actin, DAPI and KI67 (proliferative cells). Maximum intensity projection of the section corresponding to the pillar (left) and to the base (middle). Orthogonal section of the whole villus axis (right). Scale bars: 100 $\mu \mathrm{m}$. (B) Representative confocal images of Uniform (top) and Asymmetric (bottom) samples at day 3 of culture immunostained for F-actin and CK20 (differentiated cells). Maximum intensity projection of the section corresponding to the pillar (left) and to the base (middle). Orthogonal section of the whole villus axis (right). Scale bars: $100 \mu \mathrm{m}$. (C) Graph showing the frequency of $\mathrm{KI} 67^{+}$cells at day 3 of culture at the base and the tip of the pillars with respect to the total $\mathrm{KI} 67^{+}$cells under ISC-niche biomolecules gradients 
(Asymmetric) or no gradients (Uniform) (left). $\mathrm{N} \geq 3$, pillars $\geq 19$. P-value: $* * *<0.001$. Mean \pm SEM. Quantification of the relative change of $\mathrm{Ki}^{+} 7^{+}$cells from Day 1 to Day 3 at the base and pillar sections for Uniform and Asymmetric (middle). $\mathrm{N} \geq 1$, pillars $\geq 3$. P-value: $*<0.05$. Mean \pm SD. Normalized mean CK20 signal along the rescaled villus axis in Asymmetric or Uniform conditions at day 3 of culture (right). $\mathrm{N} \geq 3$, pillars $\geq 19$. Mean \pm SEM.

When we looked at the distribution of the proliferative cells under different gradients, we observed that the frequency of the proliferative cells significantly increased at the base compared to the tip from day 1 to day 3 under the Asymmetric condition, while it stayed unchanged at the base and the tip for Asymmetric 2.0 (Figure $5 \mathrm{C}$ bottom). This finding suggests that, in Asymmetric 2.0 condition, the whole range of concentrations of the ISC factors from the base to the tip of the microstructures is high enough to maintain all the cells at proliferative state. However, in the Asymmetric condition, only the concentrations present at the base allow the cells to stay proliferative. Under Asymmetric Wnt2b condition, the frequency of the proliferative cells found at the base increased from day 1 to day 3 compared to Asymmetric 2.0, presumably due to replacement of ISEMF_CM for Wnt2b (Figure $5 \mathrm{C}$ bottom). On the other hand, the distribution of proliferative cells was not significantly different between the base and the tip, in line with our results for Asymmetric 2.0 condition. Altogether, these findings demonstrate that different ISC niche biochemical gradients lead to changes in cell type composition and distribution in our villus-like 3D intestinal epithelial model, proving it useful for the study of such gradients on the cellular response. 


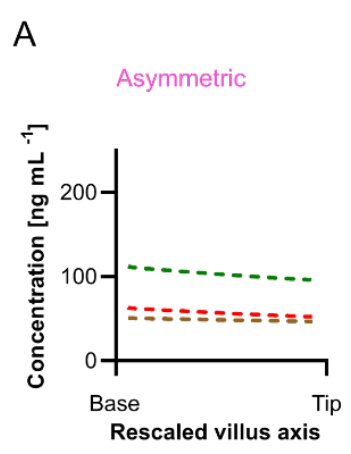

$\mathrm{B}$
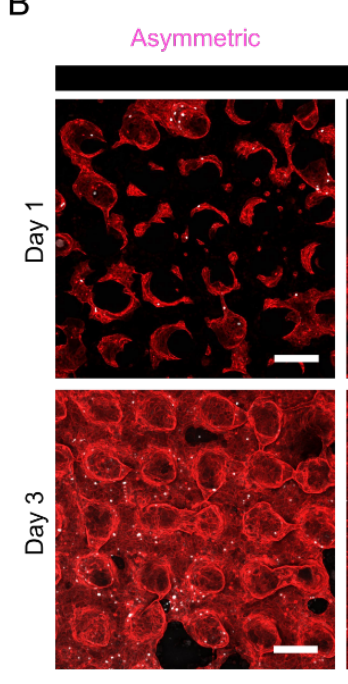

Asymmetric 2.0

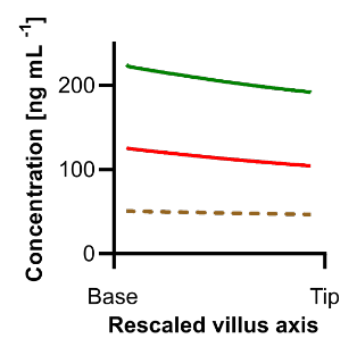

Asymmetric Wnt2b

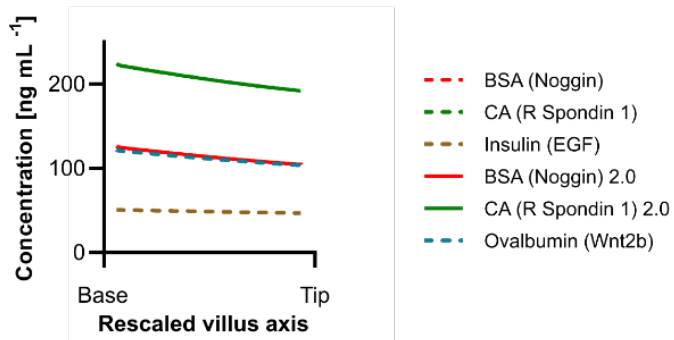

Figure 5. (A) Graphs showing the change in concentrations as a function of position along the villus axis predicted by the FEM simulations at $24 \mathrm{~h}$ for BSA (Noggin), BSA 2.0 (doubled source concentration), CA (R Spondin 1), CA 2.0 (doubled source concentration), Insulin (EGF) and Ovalbumin (Wnt2b) present in the corresponding Asymmetric (left), Asymmetric 2.0 (middle), Asymmetric Wnt2b (right) culture conditions. (B) Representative confocal maximum intensity projections images of Asymmetric, Asymmetric 2.0 and Asymmetric Wnt2b samples at days 1 and 3 of cell culture immunostained for F-actin and Ki67 (left). Scale bars: $200 \mu \mathrm{m}$. (C) Quantification of the percentage of $\mathrm{Ki}^{+} 7^{+}$(proliferative) cells with respect to the total number of cells for each culture condition (top). $N \geq 1$, pillars $\geq 5$. Mean \pm SEM. Quantification of the relative change of $\mathrm{Ki}^{+} 7^{+}$cells from Day 1 to Day 3 at the base and pillar sections for each culture condition (bottom). $\mathrm{N} \geq 1$, pillars $\geq 3$. P-value: $*<0.05$. Mean $\pm \mathrm{SD}$. 


\section{Discussion}

Biomolecular gradients present along the crypt-villus axis of the intestinal epithelium play a key role in the tissue organization and homeostasis by driving the maintenance of the intestinal stem cells (ISCs) at the crypts and their ordered differentiation along the villus axis. ${ }^{[46]}$ The characteristic $3 \mathrm{D}$ architecture of the intestinal epithelium is fundamental for the formation and maintenance of such gradients in vivo. ${ }^{[19]}$ Still, intestinal organoids are able to self-organize into crypt and villus-like regions, ${ }^{[14]}$ as well the organoid-derived 2D monolayer models, ${ }^{[47,48]}$ which underlines the capacity of epithelial-derived gradients, mainly orchestrated by Paneth cells, ${ }^{[7,13,49]}$ to organize the tissue. However, the gradients formed by biochemical factors such as Noggin and Gremlin, ${ }^{[12]}$ R-Spondins ${ }^{[50]}$ and $\mathrm{Wnt}^{2 \mathrm{~b}^{[9]}}$ secreted by intestinal subepithelial myofibroblasts (ISEMFs) located below the crypts are also needed for ISC maintenance and differentiation. Since biomolecular gradients are spatially patterned through the crypt-villus axis, in vitro models need to provide a crosstalk between the architectural cues and the biomolecular gradients to induce tissue patterning and thus be physiologically relevant. Intestinal organoids that, otherwise, show stochastic growth with crypt buddings variable in number, size, and orientation, can be patterned in a predefined manner by providing cells with architectural ${ }^{[21,24,51]}$ and biochemical ${ }^{[21,22]}$ cues using microengineered systems. Here, we used an in-house developed photolithographybased approach to produce $3 \mathrm{D}$ villus-like hydrogels ${ }^{[27]}$ to provide cells with topographical cues with well-controlled biochemical gradients, as characterized by the state-of-the-art light sheet fluorescence microscopy and the in-silico modelling. As opposed to collagen scaffolds which shrink with time and whose fabrication through replica molding requires multiple steps and the use of intermediate molds, ${ }^{[21,24]}$ the simple and moldless fabrication procedure that we use here enables to routinely incorporate $3 \mathrm{D}$ architectural cues in cell culture systems like Transwell, which is at the same time an ideal tool to generate biochemical gradients as it provides two separate basolateral and apical compartments. Using our platform, we showed that the presence of biochemical gradients of the ISC niche, mainly those generated by ISEMFs, was necessary for an in vivo-like compartmentalization of proliferative and differentiated cells. Similar results were reported when a 3D crypt-villus architecture was combined with gradients of Wnt3a, RSpondin 3 and Noggin, ${ }^{[21]}$ or when ISEMFs were embedded within the crypt-villus microstructured hydrogels. ${ }^{[24]}$ These findings point out the importance of recapitulating 
in vitro the gradients of the factors secreted by ISEMFs for an in vivo-like tissue organization. When we modified the gradient profiles by increasing concentrations or by adding new factors like Wnt2b, we no longer observed cell compartmentalization. We hypothesize that this loss of compartmentalization might be due to the higher concentrations that cells were receiving, as the gradients of ISC niche factors, which are only active in the crypt compartment in vivo, ${ }^{[46]}$ were spanning the entire vertical axis of the scaffolds. Using our system, one can determine which ISC niche components and their optimal source concentrations are needed to obtain in vivo-like tissue compartmentalization. We believe that the culture platform that we present here, with physiologically representative and well-controlled microenvironment (3D architecture and gradients of ISC biochemical factors), will be useful to systematically test the effect of a wide range of ISC niche factors in the context of understanding intestinal tissue dynamics.

\section{Experimental Section}

Fabrication of villus-like microstructured hydrogels: The villus-like microstructured hydrogels were fabricated by photolithography, as previously described. ${ }^{[27]}$ Briefly, a prepolymer solution containing PEGDA (6.5\% (w/v), $6 \mathrm{kDa}$ of MW, Sigma-Aldrich), acrylic acid (AA) $(0.3 \%$ (w/v), Sigma-Aldrich), and Irgacure D-2959 photoinitiator (1\% (w/v), Sigma-Aldrich) in phosphate-buffered saline (PBS) was flown into a chip fabricated with a $1 \mathrm{~mm}$ thick polydimethylsiloxane (PDMS) (Sylgard 184, Dow Corning) stencil containing an array of pools of $6.5 \mathrm{~mm}$ diameter. Tracketch polyethylene terephthalate (PET) membranes of $5 \mu \mathrm{m}$ pore size (Sabeu GmbH \& Co.) were used as substrates for the hydrogels. The microstructured PEGDA-AA hydrogels were fabricated by exposing the pre-polymer solution to UV light under patterned photomasks with transparent windows of $150 \mu \mathrm{m}$ in diameter and a density of 25 windows $\mathrm{mm}^{-2}$ (designed by Inkscape software and printed on acetate films (CAD/Art Services)). The photolithography was performed in an MJBA mask aligner (SUSS MicroTech) using a power density of $25 \mathrm{~mW} \mathrm{~cm}^{-2}$. The prepolymer solution was exposed for $\approx 220 \mathrm{~s}$ to form the villus-like micropillars (Figure $1 \mathrm{~A}$ ). A second exposure of $\approx 25 \mathrm{~s}$ was performed to form a hydrogel base holding the microstructures together. After UV exposure, unreacted polymer and photoinitiator were washed out with PBS and the hydrogels were kept 
submerged at $4^{\circ} \mathrm{C}$ for at least 3 days to reach equilibrium swelling. Samples fabricated onto PET membranes were then assembled on modified Transwell inserts or microfluidic chips using double-sided pressure-sensitive adhesive (PSA) rings (Adhesive Research) as detailed previously. ${ }^{[27]}$ After swelling, and to provide the scaffolds with cell-adhesion motifs, the PEGDA-AA hydrogels were functionalized with collagen type I $(10 \% \mathrm{w} / \mathrm{v}$ or $0.4 \mathrm{mg} \mathrm{mL}^{-1}$, Sigma-Aldrich) via N - (3-Dimethylaminopropyl)-N ' - ethylcarbodiimide (EDC)/N-Hydroxysuccinimide (NHS) (Sigma-Aldrich) mediated coupling. Right after functionalization, the samples were used for cell culture experiments.

Characterization of the mesh size of PEGDA-AA hydrogels: The mesh size of PEGDAAA hydrogels was estimated using Flory-Rehner equilibrium swelling theory as modified by Peppas and Merrill for hydrogels diluted in aqueous media. ${ }^{[35,36]}$ This was an essential parameter to assess the diffusivity of biomolecules through the hydrogels. Hydrogels were produced from pre-polymer solutions containing PEGDA (5 - $10 \%$ (w/v)), I2959 $(1 \%(\mathrm{w} / \mathrm{v}))$ and AA $(0.06 \%(\mathrm{w} / \mathrm{v}))$ in PBS and were casted as discs, $10 \mathrm{~mm}$ in diameter and $\approx 1 \mathrm{~mm}$ in height. Note that changes in AA concentration from $0.06 \%$ to $0.6 \% \mathrm{w} / \mathrm{v}$ don't have any significant effects on the swelling behavior of the hydrogels. ${ }^{[27]}$ Hydrogels were fabricated using a power density of $25 \mathrm{~mW} \mathrm{~cm}^{-2}$ and $150 \mathrm{~s}$ and $200 \mathrm{~s}$ of UV exposure. The weight of the PEGDA-AA hydrogels was then measured at three different time points, i.e. after fabrication (also called after curing, $\mathrm{m}_{\mathrm{c}}$ ), at equilibrium swelling (swollen in PBS at $4^{\circ} \mathrm{C}$, up to 7 days, $\mathrm{m}_{\mathrm{s}}$ ), and at dry state (dried till constant weight, $\mathrm{m}_{\mathrm{d}}$ ). Once these values were obtained, the mesh size was calculated using previously established equations. ${ }^{[52]}$ For the detailed explanation of the calculations and the equations used, see Supporting Information.

Fabrication of a microfluidic chip for biomolecule gradient generation and visualization: A PDMS chip for the generation and visualization of biomolecular gradients through the hydrogel network was designed and fabricated. It contained a microfluidic channel and a chamber allocating individual hydrogels (Figure 2A, left). For the fabrication of the microfluidic channel, a mold was prepared using SU-8 resist on a silicon wafer ( $\mathrm{Si}(111)$, 1-side polished, n-type) (MicroChemicals) and photolithography. The wafer was sequentially cleaned with acetone and isopropanol, and oxygen plasma cleaning (PDC002, Harrick Scientific) for $20 \mathrm{~min}$ at low RF $(6.8 \mathrm{~W})$. After drying (hot plate at $\left.95^{\circ} \mathrm{C}\right)$ to remove the excess of humidity, a $100 \mu \mathrm{m}$ thick layer of SU-8 2100 negative photoresist 
(Microchem) was spun-coated (WS-650MZ- 23NPP/LITE, Laurell Technologies) on the wafer. The film was baked for $5 \mathrm{~min}$ at $65^{\circ} \mathrm{C}$ and $20 \mathrm{~min}$ at $95^{\circ} \mathrm{C}$ to evaporate the solvent and it was exposed to UV light $\left(25 \mathrm{~mW} \mathrm{~cm}^{-2}\right)$ through a photomask with the channel design (in a rectangular transparent window, $200 \mu \mathrm{m}$ in width and $20 \mathrm{~mm}$ in length). After a post-bake $\left(5 \mathrm{~min}\right.$ at $65^{\circ} \mathrm{C}$ and $10 \mathrm{~min}$ at $95^{\circ} \mathrm{C}$ ), the SU-8 film was developed (Microchem developer), rinsed with isopropanol, and dried under nitrogen. Right after fabrication, the height of the channel was measured by profilometer (DEKTAK 6M, Veeco Instruments) as $104.3 \pm 0.1 \mu \mathrm{m}$. To avoid sticking when producing PDMS replicas, SU-8 molds were silanized with (Tridecafluoro-1,1,2,2-tetrahydrooctyl)trichlorosilane (ABCR GmbH \& Co. KG), in vapor phase. Then, PDMS replicas containing the microfluidic channel were punched with a $0.75 \mathrm{~mm}$ punch (Harris Uni-Core, GE Healthcare Life Sciences) at each end of the channel, and with a $4 \mathrm{~mm}$ punch at the center to form a chamber below the hydrogel (Figure S2 A). Afterwards, they were plasma bonded to a $1 \mathrm{~mm}$ thick PDMS slab to close the microchannel and leave an open chamber to allocate the villus-like microstructured hydrogel. The hydrogel was bonded using double-sided PSA rings. The microchannel and the bottom chamber were filled with PBS and prior to use, PBS was exchanged for the corresponding protein solution prepared in PBS- $0.1 \%$ Tween 20.

In-chip diffusion studies on PEGDA-AA hydrogels using light-sheet fluorescence microscopy (LSFM): A custom-made light-sheet microscope was developed for the visualization of the biomolecular gradients formed through the hydrogel network when allocated within the chip. Continuous wave lasers with wavelengths of $488 \mathrm{~nm}$ (MLD 50 $\mathrm{mW}$, Cobolt) and $561 \mathrm{~nm}$ (DPL $100 \mathrm{~mW}$, Cobolt) were used for fluorescence excitation. The light sheet was created by a pair of galvanometric mirrors (GVSM002, Thorlabs) conjugated with the illumination objectives (Plan-Fluor 4x, NA: 0.13, Nikon) through a pair of achromatic doublets (AC254-050-A- ML ( $\mathrm{f}=50 \mathrm{~mm})$ and AC254-200-A-ML ( $\mathrm{f}=$ $200 \mathrm{~mm}$ ), Thorlabs), creating a flat top Gaussian beam profile to assure its homogeneity. ${ }^{[53]}$ The detection was performed in an up-right configuration, using water dipping objectives (PlanFluor 10x, NA: 0.3, Nikon) (Figure 2A, right). An achromatic doublet (AC254- 200-A-ML, Thorlabs) formed an image onto a Hamamatsu Orca Flash 4.0 CMOS camera chip. Emission filters of 520/15 (for autofluorescence) and 620/52 (for Texas red) (Semrock) were selected using a motorized filter wheel (FW102C, Thorlabs). To be able to perform in-chip measurements, a special sample holder was designed using FreeCAD software and 3D printed (Hephestos 2, BQ) using poly(lactic acid) (PLA) as 
printing material (Figure 2A, right). It consisted of a square pool with top open access for the sample insertion and imaging, and two glass windows on the sides for proper lightsheet illumination. The chamber included a glass covered pedestal (oriented $25^{\circ}$ from the optical table) where the microfluidic chip allocating the hydrogel was glued (Loctite). This pedestal also allowed bright-field illumination with a LED for sample positioning. With this inclination, the sample was illuminated and imaged transversally, to avoid any barrier on the path of the light-sheet. Laser scanning for imaging was performed by vertical translation of the sample holder using a motorized stage (PI M-501.1DG, Physik Instrumente), through a fixed horizontal light-sheet plane. All the components of the microscope were controlled using a custom-made software (LabView).

Biomolecular gradients were then formed by filling in the source compartment of the chip through the microfluidic channel through the inlet port while the hydrogel was in the allocated position. Biomolecular gradients formed by diffusing bovine serum albumin (BSA) protein fluorescently labelled with Texas Red (BSA_TxRED, Thermo Fisher) within the PEGDA-AA hydrogel networks were imaged by performing a time-lapse acquisition of a 3D volume of $1.33 \times 1.33 \times 2.3 \mathrm{~mm}$ over two hours with a frame interval of $15 \mathrm{~min}$, and a stack step of $5 \mu \mathrm{m}$. The source chip compartment was loaded with BSA_TxRED dissolved in PBS-0.1\% Tween20 at a concentration of $10 \mu \mathrm{g} \mathrm{mL}^{-1}$ and the imaging was started 3 hours after loading. To minimize data load, we binned the image by a factor of 4 . Then, the overall lateral pixel size was $2.6 \mu \mathrm{m}$. The image processing was carried out using ImageJ software. ${ }^{[54]}$ First, the stacks were resliced (ImageJ reslice function) to obtain a vertical view of the source compartment, the hydrogel and the sink chip compartment. Then, a plane in the middle of the stack where the radial diffusion could be neglected was chosen. A linear region of interest (ROI) of 10 pixels in size was drawn along the perpendicular axis of the hydrogel. The relative fluorescence intensity (RFI) values along that line were obtained for all the time points acquired. These values were converted to protein concentrations by first constructing a calibration curve. For that, the fluorescence intensity of solutions with different BSA_TxRED concentrations $\left(0-100 \mu \mathrm{g} \mathrm{mL}^{-1}\right)$ were measured using the same LSFM set-up. For each concentration 21 measurements were made. Then, ROIs of $200 \times 150$ pixels in size were selected at the center of the stacks acquired in each measurement and the mean fluorescence intensity values were calculated. The background intensity value was determined from blank PBS. 
In-silico modelling of protein diffusion through PEGDA-AA hydrogels: In order to be able to estimate the spatio-temporal gradients of our molecules of interest (epidermal growth factor (EGF), R-Spondin 1, Noggin and Wnt2b) produced by the diffusion chip on the villus-like microstructured PEGDA-AA hydrogels, an in-silico model was developed. For that, finite element method (FEM) was employed. First, the diffusion coefficient of the proteins in an aqueous solution, $\mathrm{D}_{0}$, was calculated using Stokes-Einstein equation (Equation 1).

$D_{0}=\frac{k_{b} T}{3 p h d_{h}}$

where $\mathrm{k}_{\mathrm{b}}$ is the Boltzmann's constant $\left(1.38 \times 10^{-23} \mathrm{~J} \mathrm{~K}^{-1}\right), T$ is the absolute temperature $\left(37^{\circ} \mathrm{C}=310.15 \mathrm{~K}\right), \eta$ is the viscosity of the solvent $\left(6.915 \times 10^{-4} \mathrm{~N} \mathrm{~m} \mathrm{~s}^{-2}\right.$ for water at $37^{\circ} \mathrm{C}^{[55]}$ ) and $\mathrm{d}_{\mathrm{h}}$ is the protein hydrodynamic diameter. As $\mathrm{d}_{\mathrm{h}}$ was not available in literature for our proteins of interest, for FEM simulations, the reported values of $d_{h}$ corresponding to proteins with comparable sizes were selected (Table 1).

Table 1: ISC niche biochemical factors and their corresponding model proteins with their molecular mass and the hydrodynamic diameters of the model proteins.

\begin{tabular}{lllll}
\hline ISC niche factor & MW [kDa] & Model protein & MW [kDa] & $\mathrm{d}_{\mathrm{h}}[\mathrm{nm}]$ \\
\hline EGF & 6.0 & Insulin & 5.7 & $2.94^{[55]}$ \\
R-Spondin 1 & 25.6 & $\mathrm{CA}^{\mathrm{a})}$ & 29.0 & $4.70^{[55]}$ \\
Noggin & 46.4 & $\mathrm{BSA}$ & 66.5 & $7.20^{[41]}$ \\
Wnt2b & 38.0 & Ovalbumin & 43.0 & $5.96^{[55]}$ \\
\hline
\end{tabular}

a) CA: Carbonic anhydrase.

The diffusion of proteins over time and space was described by Fick's second law and simulated employing the 'Transport of diluted species' module of COMSOL Multiphysics software (version 5.5). 3D forms were reduced to 2D geometries assuming axial symmetry. The geometry was composed of two media: the PEGDA-AA hydrogel and the aqueous solution. For each medium the corresponding diffusion coefficient values of each species (i.e. $\mathrm{D}_{0}$ for an aqueous solution, and $\mathrm{D}_{\mathrm{g}}$ for a PEGDA-AA hydrogel network) used are listed in Table 2. $\mathrm{D}_{0}$ values were calculated using Stokes-Einstein equation and $d_{h}$ values from Table $1 . D_{g}$ values were assumed to be close to those reported in literature for model proteins diffusing through PEGDA-based hydrogels with similar network structures (PEGDA concentrations and chain length). 
Table 2: For each species, diffusion coefficients values at $37^{\circ} \mathrm{C}$ in the aqueous solution $\left(D_{0}\right)$ and in the PEGDA hydrogel $\left(D_{g}\right)$; the source concentrations used.

\begin{tabular}{lllll}
\hline $\begin{array}{l}\text { Model } \\
\text { protein }\end{array}$ & $\mathrm{D}_{0} \cdot 10^{-10}\left[\mathrm{~m}^{2} \cdot \mathrm{s}^{-1}\right]$ & $\mathrm{D}_{\mathrm{g}} \cdot 10^{-10}\left[\mathrm{~m}^{2} \cdot \mathrm{s}^{-1}\right]$ & Concentration $\left[\mathrm{ng} \cdot \mathrm{mL}^{-1}\right]$ & Molarity $\cdot 10^{-6}\left[\mathrm{~mol} \cdot \mathrm{m}^{-3}\right]$ \\
\hline Insulin & 2.23 & $1.27^{[55]}$ & 300 & 52.6 \\
CA & 1.40 & $0.16^{[55]}$ & 600 & 20.7 \\
BSA & 0.91 & $0.13^{[41]}$ & 300 & 4.5 \\
Ovalbumin & 1.10 & $0.20^{[55]}$ & 600 & 14.0 \\
\hline
\end{tabular}

As in the experimental setup, we modeled the gradients formation in a system composed by a source, the hydrogel, and a sink compartment. The initial concentration was set to zero in the entire geometry except for that of the source. The biomolecule concentrations commonly used in a standard organoid culture were tripled for the source (Table 2) to compensate for the biomolecules trapped at the membrane $(\approx 66 \%)$, as determined in preliminary experiments (data not shown). Note that, even though the mass concentrations are similar or equal, the molarities are different due to differences in protein size. No flux boundary was imposed for the rest of the the edges of the geometry. The villus-like microstructures were implemented in the simulation as an array of semi ellipses with a semi-minor axis of $75 \mu \mathrm{m}$ (villus width) and a semi-major axis of $350 \mu \mathrm{m}$ (villus height). The center-to-center spacing was set to $250 \mu \mathrm{m}$. The array was considered to be on top of a hydrogel base $150 \mu \mathrm{m}$ thick (Figure 2B, left at the lower row). The sink medium was represented as a rectangular form. The FEM mesh for the aqueous medium was set to "finer" $\left(10^{-2} \mathrm{~mm}<\right.$ FEsize $\left.<1 \mathrm{~mm}\right)$ and the mesh for the hydrogel medium was set to "extra fine" $\left(10^{-3} \mathrm{~mm}<\right.$ FEsize $\left.<2 \cdot 10^{-2} \mathrm{~mm}\right)$. To validate the simulations with the experimental data, BSA diffusion coefficients (in accordance with LSFM experiments) and a source concentration of $10 \mu \mathrm{g} \mathrm{mL}^{-1}$ were used. To fully capture experimental fluorescence profiles, a thin diffusion barrier between the source and the hydrogel was added to simulate the protein accumulation observed at the PET membrane ( $D=5 \times 10^{-}$ $\left.{ }^{14} \mathrm{~m}^{2} \mathrm{~s}^{-1}\right)$. For the simulations emulating the gradient visualization experiments performed at RT using LSFM, $\mathrm{D}_{0}$ and $\mathrm{D}_{\mathrm{g}}$ values for BSA at $25^{\circ} \mathrm{C}$ were used $\left(\mathrm{D}_{0}\right.$ at $25^{\circ} \mathrm{C}=6.8 \times 10^{-}$ ${ }^{11} \mathrm{~m}^{2} \mathrm{~s}^{-1}$ and $\mathrm{D}_{\mathrm{g}}$ at $25^{\circ} \mathrm{C}=1.0 \times 10^{-11} \mathrm{~m}^{2} \mathrm{~s}^{-1[41]}$ ). This in-silico model was then used to predict the spatio-temporal gradient profiles of the molecules of interest (EGF, R Spondin 1, Noggin, and Wnt2b) formed on the villus-like microstructured hydrogels. Note that, as the cell culture was performed in a Transwell culture setup (see ahead), the source and the sink dimensions were adjusted accordingly. The diffusion coefficients and source 
concentrations detailed in Table 2 were employed for the simulations. The simulations were performed up to $24 \mathrm{~h}$ of diffusion time, which was found to be a convenient frequency for the cell culture medium exchange and also it was confirmed with our simulations that gradients profiles could be stabilized by this periodic medium replenishment approach (see Supporting Information for details). As results, the factor concentration profiles as a function of the vertical position (z) along a line spanning the source, the hydrogel and the sink (Figure 2B, bottom left), and also along the curve contouring the villus-like surface were computed at different time intervals (see the corresponding figure legends) and the graphs were plotted using GraphPad software. Base to tip decrease in biomolecular concentrations was calculated using the concentration values computed at the base and the tip for each factor.

Mouse model: Lgr5-EGFP-IRES-creERT2 mice, previously described, ${ }^{[3]}$ were used. Briefly, Lgr5-EGFP-IRES-creERT2 mice were generated by homologous recombination in embryonic stem cells targeting the EGFP-IRES-creERT2 cassette to the ATG codon of the stem cell marker Lgr5 locus, allowing the visualization of $\operatorname{Lgr} 5^{+}$stem cells with a green fluorescent protein (GFP). All experimental protocols involving mice were approved by the Animal care and Use Committee of Barcelona Science Park (CEEAPCB) and the Catalan government and performed in accordance with their relevant guidelines and regulations.

Intestinal crypt isolation and culture: Mice intestinal crypts were isolated as previously described. ${ }^{[56,57]}$ In short, small intestines were washed with PBS and cut longitudinally. Villi were mechanically removed, and intestinal crypts were extracted by incubating the tissue with $2 \mathrm{mM}$ of EDTA (Sigma-Aldrich) in PBS for 40 minutes at $4^{\circ} \mathrm{C}$. The digestion content was filtered through a series of $70 \mu \mathrm{m}$ pore cell strainers (Biologix Research Co.) to obtain the crypt fractions. Crypts were resuspended in Matrigel (BD Bioscience) drops and cultured with basic organoid growth medium (Advanced DMEM/F12 (Invitrogen), Glutamax (1\%, Gibco), HEPES (1\%, Sigma-Aldrich), Normocin (1:500, Invitrogen), B27 (2\%, Gibco), N2 (1\%, Gibco), N-acetylcysteine (1.25 $\mathrm{mM}$, Sigma-Aldrich)), supplemented with recombinant murine EGF (100 ng mL-1, Gibco), recombinant human R-spondin 1 (200 ng mL-1, R\&D Biosystems), recombinant murine Noggin (100 ng mL ${ }^{-1}$, Peprotech), CHIR99021 (3 $\mu \mathrm{M}$, Tebu-bio) and valproic acid (1 mM, Sigma-Aldrich) to obtain ENRCV medium. The first 4 days of culture the Rho kinase inhibitor Y-27632 (Sigma-Aldrich) was added. The medium was changed 
every 2 to 3 days. Outgrowing crypts were passaged twice a week and organoid stocks were maintained for up to 4 months.

Intestinal subepithelial myofibroblast conditioned medium (ISEMF_CM) preparation: Intestinal subepithelial myofibroblasts (ISEMFs) were isolated from mice intestines following a previously reported protocol. ${ }^{[58]}$ Briefly, left over tissue from the crypt isolation procedure was further digested with $2000 \mathrm{U}$ of collagenase (Sigma-Aldrich) for 30 minutes at $37^{\circ} \mathrm{C}$. The digested tissue was centrifuged and the pellet was resuspended in DMEM, high glucose, GlutaMAX TM, pyruvate (Life Technologies) containing fetal bovine serum (FBS) (10\%, Gibco), penicillin/streptomycin (1\%, Sigma-Aldrich), and minimal essential medium non-essential amino acids (MEM-NEAA) (1\%, Gibco) and cultured in tissue culture plates. After 1 week in culture, lamina propria myofibroblasts positive for $\alpha$-smooth muscle actin ( $\alpha$-SMA) and vimentin and negative for desmin (data not shown) remained attached. ${ }^{[10]}$ Plates at 60 to $80 \%$ of confluence were maintained in culture for 6 days and then the supernatant was collected and complemented with B27 $(2 \%), \mathrm{N} 2(1 \%)$ and $\mathrm{N}$-acetylcysteine $(1.25 \mathrm{mM})$ to obtain what we called intestinal subepithelial myofibroblast conditioned medium (ISEMF_CM).

Formation of biomolecular gradients along the vertical axis of the villus-like microstructured PEGDA-AA hydrogels: Gradients of the biomolecules reported to be relevant for the formation and maintenance of the intestinal stem cell (ISC) niche at the intestinal crypts, listed in Table 1, were established as predicted by the in-silico model. For that purpose, villus-like microstructured hydrogels fabricated on top of PET membranes were mounted on Transwell inserts, which allow the use of different solutions at the apical and basolateral compartments. To account for the cellular effects of the biomolecular gradients formed, four different culture conditions were established: (i) asymmetric, where ENRCV and ISEMF_CM were delivered basolaterally and basal medium was delivered apically; (ii) uniform, with the basolateral and apical delivery of ENRCV and ISEMF_CM; (iii) asymmetric 2.0, where the concentrations of Noggin and R-spondin were doubled compared to asymmetric while keeping basal medium at the apical chamber, and (iv) asymmetric Wnt2b, where the ISEMF_CM was replaced by the recombinant protein $\mathrm{Wnt} 2 \mathrm{~b}$, so ENRCV and $\mathrm{Wnt} 2 \mathrm{~b}$ were delivered in the basolateral chamber and basal medium at the apical side. Final concentrations for the different relevant biomolecules (Table 2) were determined from the simulations and are detailed at Table S1. Notice that, in order to compensate for $\approx 66 \%$ of protein concentration getting 
trapped at the membrane, protein source concentrations were tripled compared to concentrations used for organoids culture within Matrigel drops. For all four conditions, the different media were introduced in the corresponding Transwell compartment 24 hours before cell seeding and, according to the simulation data, we renewed the medium of both compartments every 24 hours to maintain the gradients formed.

Organoid-derived cell culture onto villus-like PEGDA-AA microstructured hydrogels with biomolecular gradients: Matrigel drops containing fully-grown organoids were shattered by pipetting with TrypLE Express1X (Gibco) and passed several times through a syringe with a $23 \mathrm{G}$ needle (BD Microlance 3). Next, disrupted organoids were further digested to single cells by incubating them for 5 to 7 minutes at $37^{\circ} \mathrm{C}$ with vigorous handshaking every minute. Successful digestion to single cells was confirmed via inspection under the microscope. Prior to cell seeding on the villus-like microstructured hydrogels, the media of both apical and basolateral compartments of the Transwells were renewed to maintain the established gradients. Then, $5 \times 10^{5}$ intestinal epithelial cells were resuspended in $100 \mu \mathrm{L}$ of the corresponding apical medium and seeded on the hydrogels. Once the cells were adhered (after 1 hour), $100 \mu \mathrm{L}$ of the corresponding apical medium were added. Cell cultures were maintained in an incubator at $37^{\circ} \mathrm{C}$ and $5 \% \mathrm{CO}_{2}$ for at least one day.

Immunostaining: Cells were fixed with 10\% neutralized formalin (Sigma-Aldrich), permeabilized with 0.5\% Triton X-100 (Sigma-Aldrich), and blocked with a blocking buffer containing 1\% BSA (Sigma-Aldrich), 3\% donkey serum (Millipore), and 0.2\% Triton X-100 in PBS for at least 2 hours at room temperature (RT). The primary antibodies used were: anti-Ki67 (1:100, Abcam), anti-Ki67 (1:100, BD Biosciences) and anti-cytokeratin 20 (1:100, Dako). All samples were incubated with the primary antibody overnight at $4{ }^{\circ} \mathrm{C}$ followed by $1 \mathrm{~h}$ incubation at RT with secondary antibodies, Alexa Fluor 568 and Alexa Fluor 488 donkey anti-goat, and Alexa Fluor 647 (Jackson ImmunoResearch) diluted at 1:500. Nuclei were stained with 4',6-diamidino-2phenylindole (DAPI) (1:1000, Thermo Fisher Scientific). Alexa Fluor 568 phalloidin was used to stain filamentous actin (F-actin) (3.5:500, Cytoskeleton).

Image acquisition and analysis: Fluorescence images were acquired at randomly selected locations using a confocal laser scanning microscope (LSM 800, Zeiss) with a 10x air objective $(\mathrm{NA}=0.3, \mathrm{WD}=2.0)$. The laser excitation and emission light spectral collection were optimized for each fluorophore, especially for the four-color scans, where 
the emission bands were carefully adjusted to avoid overlapping channels. The pinhole diameter was set to 1 Airy Unit (AU). A z-step of $5 \mu \mathrm{m}$ was used. The relative coverage was quantified by measuring the cell area after thresholding with Image J software the maximum intensity projected F-actin fluorescence images, and normalizing to surface of the substrate (base + pillars). For this, we employed a model to estimate the effective surface of the substrate from the projected image (see Supporting Information for the details). In addition, confocal fluorescence microscopy images of Ki67 and CK20 markers were used to quantify the proliferative and differentiated cell populations respectively, along the vertical axis of the villus-like microstructures. For this, a custommade Image J macro was used to divide the z confocal stacks to tiles, each comprising one single villus. Then, each covered villus was analyzed using IMARIS (BitPlane, version 9.1.0). As a pre-processing step, we applied a mean filter of $3 \times 3 \times 3$ px to the Ki67 channel to reduce the dotty unspecific staining of this antibody. Then, nuclei were detected with the Imaris Spot detector (diameter set to $9 \mu \mathrm{m}$ ) by adjusting the threshold manually for optimal results. Next, Ki67 positive cells were identified by measuring the mean intensity around the positions of the detected nuclei and setting a minimum threshold for positive cells. Finally, we exported the $\mathrm{Z}$ positions of all cells and of Ki67+ cells. For each tile, $\mathrm{Z}$ values were rescaled from 0 to 1,0 corresponding to the nucleus detected closer to the villus base and 1 to the nucleus closer to the villus tips. $Z$ was divided into five equal segments for data interpretation purposes. Next, we estimated the percentage of $\mathrm{Ki}^{+} 7^{+}$cells with respect to the total number of cells for the five regular $\mathrm{Z}$ segments along each villus. Finally, we computed the fractioned percentage of each segment. To quantify the relative change in the fequency of $\mathrm{Ki} 67^{+}$cells from day one to day three, we subtracted the mean frequency of Ki67 $7^{+}$cells of day 1 to that of day 3 and we normalized the value by the mean frequency of day 1 . To quantify the CK20 intensity signal along the vertical axis of each villus we used another custom-made ImageJ macro. Briefly, we run the ImageJ built-in plot profile function within a drawn ROI surrounding the villus to quantify mean intensity for the CK20 and F-acting fluorescent channels and we exported the data. We normalized the CK20 intensity values by the F-actin ones and we rescaled them from 0 to 1 to eliminate the intensity variations due to the image acquisition set up. Then, we rescaled the villus vertical axis from 0 to 1 and divided it into five equal segments for data interpretation purposes. Finally, we computed the mean intensity fraction of each segment. 
Statistical analysis: $\mathrm{N}$ is the number of independent experiments and $\mathrm{n}$ is the number of samples. The data were presented in the figures as mean \pm error (standard error of the mean (SEM) or standard deviation (SD) as indicated in the figure captions). Statistical comparisons were performed using two-tailed, unequal variance t-test and a $\mathrm{p}<0.05$ was considered to be significant.

\section{Supporting Information}

Supporting Information is available from the Wiley Online Library or from the author.

\section{Acknowledgements}

We would like to thank Rene Fabregas for the advice on the in-silico model. We also thank Jordi Comelles, María García and Enara Larrañaga for the fruitful discussions. Funding for this project was provided by European Union's Horizon 2020 ERC grant agreements No 647863 (COMIET) and No 884623 (residualCRC), the CERCA Programme/Generalitat de Catalunya (2017-SGR-1079 and 2017-SGR-698), LaserlabEurope EU-H2020 grant agreement No 871124, the Spanish Ministry of Economy and Competitiveness (Severo Ochoa Program for Centers of Excellence in R\&D 2016-2019 and CEX2019-000910-S), the Spanish Ministry of Science (PID2020-119917RB-I00) and also by the Fundació Privada Cellex and the Fundació Mir-Puig. The authors would like to thank Barcelona Institute of Science and Technology (BIST) for the funding of ENGUT project through Ignite Programme. The authors gratefully acknowledge the Agència de Gestió d'Ajuts Universitaris i de Recerca (AGAUR) for the funding of G. A. through FI-DGR 2014 and to MINECO/FEDER Ramón y Cajal program (RYC-201517935) for the funding of E. G. The results presented here reflect only the views of the authors; the European Commission is not responsible for any use that may be made of the information it contains.

Received: ((will be filled in by the editorial staff))

Revised: ((will be filled in by the editorial staff))

Published online: ((will be filled in by the editorial staff))

References

[1] H. Clevers, Cell 2013, 154, 274. 
[2] M. Leushacke, N. Barker, Gut 2014, 63, 1345.

[3] N. Barker, J. H. Van Es, J. Kuipers, P. Kujala, M. Van Den Born, M. Cozijnsen, A. Haegebarth, J. Korving, H. Begthel, P. J. Peters, H. Clevers, Nature 2007, 449, 1003.

[4] P. De Santa Barbara, G. R. Van Den Brink, D. J. Roberts, Cell. Mol. Life Sci. 2003, $60,1322$.

[5] T. Sato, H. Clevers, Science (80-. ). 2013, 340, 1190.

[6] J. S. Dutton, S. S. Hinman, R. Kim, Y. Wang, N. L. Allbritton, Trends Biotechnol. 2019, 37, 744 .

[7] T. Sato, J. H. Van Es, H. J. Snippert, D. E. Stange, R. G. Vries, M. Van Den Born, N. Barker, N. F. Shroyer, M. Van De Wetering, H. Clevers, Nature 2011, 469, 415.

[8] A. Gregorieff, D. Pinto, H. Begthel, O. Destrée, M. Kielman, H. Clevers, Gastroenterology 2005, 129, 626.

[9] H. F. Farin, J. H. Van Es, H. Clevers, Gastroenterology 2012, 143, 1518.

[10] N. Y. Lei, Z. Jabaji, J. Wang, V. S. Joshi, G. J. Brinkley, H. Khalil, F. Wang, A. Jaroszewicz, M. Pellegrini, L. Li, M. Lewis, M. Stelzner, J. C. Y. Dunn, M. G. Martìn, PLoS One 2014, 9.

[11] X. C. He, J. Zhang, W. G. Tong, O. Tawfik, J. Ross, D. H. Scoville, Q. Tian, X. Zeng, X. He, L. M. Wiedemann, Y. Mishina, L. Li, Nat. Genet. 2004, 36, 111.

[12] C. Kosinski, V. S. W. Li, A. S. Y. Chan, J. Zhang, C. Ho, Y. T. Wai, L. C. Tsun, R. C. Mifflin, D. W. Powell, T. Y. Siu, Y. L. Suet, X. Chen, Proc. Natl. Acad. Sci. U. S. A. 2007, 104, 15418.

[13] H. F. Farin, I. Jordens, M. H. Mosa, O. Basak, J. Korving, D. V. F. Tauriello, K. De Punder, S. Angers, P. J. Peters, M. M. Maurice, H. Clevers, Nature 2016, 530, 340 .

[14] T. Sato, R. G. Vries, H. J. Snippert, M. Van De Wetering, N. Barker, D. E. Stange, J. H. Van Es, A. Abo, P. Kujala, P. J. Peters, H. Clevers, Nature 2009, 459, 262.

[15] A. Ootani, X. Li, E. Sangiorgi, Q. T. Ho, H. Ueno, S. Toda, H. Sugihara, K. Fujimoto, I. L. Weissman, M. R. Capecchi, C. J. Kuo, Nat. Med. 2009, 15, 701.

[16] J. R. Spence, C. N. Mayhew, S. A. Rankin, M. F. Kuhar, J. E. Vallance, K. Tolle, E. E. Hoskins, V. V. Kalinichenko, S. I. Wells, A. M. Zorn, N. F. Shroyer, J. M. Wells, Nature 2011, 470, 105.

[17] K. L. Fair, J. Colquhoun, N. R. F. Hannan, Philos. Trans. R. Soc. B Biol. Sci. 2018, 373. 
[18] M. Almeqdadi, M. D. Mana, J. Roper, Ö. H. Yilmaz, Am. J. Physiol. - Cell Physiol. 2019, 317, C405.

[19] A. E. Shyer, T. R. Huycke, C. Lee, L. Mahadevan, C. J. Tabin, Cell 2015, 161, 569.

[20] C. M. Costello, J. Hongpeng, S. Shaffiey, J. Yu, N. K. Jain, D. Hackam, J. C. March, Biotechnol. Bioeng. 2014, 111, 1222.

[21] Y. Wang, D. B. Gunasekara, M. I. Reed, M. DiSalvo, S. J. Bultman, C. E. Sims, S. T. Magness, N. L. Allbritton, Biomaterials 2017, 128, 44.

[22] Y. Wang, R. Kim, D. B. Gunasekara, M. I. Reed, M. DiSalvo, D. L. Nguyen, S. J. Bultman, C. E. Sims, S. T. Magness, N. L. Allbritton, Cmgh 2018, 5, 113.

[23] J. Creff, R. Courson, T. Mangeat, J. Foncy, S. Souleille, C. Thibault, A. Besson, L. Malaquin, Biomaterials 2019, 221.

[24] M. Verhulsel, A. Simon, M. Bernheim-Dennery, V. Ram Gannavarapu, L. Gérémie, D. Ferraro, D. Krndija, L. Talini, J.-L. Viovy, D. M. Vignjevic, S. Descroix, Lab Chip 2020.

[25] Y. Wang, D. B. Gunasekara, P. J. Attayek, M. I. Reed, M. Disalvo, D. L. Nguyen, J. S. Dutton, M. S. Lebhar, S. J. Bultman, C. E. Sims, S. T. Magness, N. L. Allbritton, ACS Biomater. Sci. Eng. 2017, 3, 2502.

[26] J. Yu, S. Peng, D. Luo, J. C. March, Biotechnol. Bioeng. 2012, 109, 2173.

[27] A. G. Castaño, M. García-Díaz, N. Torras, G. Altay, J. Comelles, E. Martínez, Biofabrication 2019, 11.

[28] A. L. Cartaxo, J. Almeida, E. J. Gualda, M. Marsal, P. Loza-Alvarez, C. Brito, I. A. Isidro, BMC Bioinformatics 2020, 21, 1.

[29] B. Abbas, T. L. Hayes, D. J. Wilson, K. E. Carr, J. Anat. 1989, 162, 263.

[30] P. Kelly, I. Menzies, R. Crane, I. Zulu, C. Nickols, R. Feakins, J. Mwansa, V. Mudenda, M. Katubulushi, S. Greenwald, M. Farthing, Am. J. Trop. Med. Hyg. 2004, 70, 412 .

[31] G. J. Tortora, B. Derrickson, Principles of Anatomy and Physiology, Vol. 53, 2008.

[32] J. Sotres, S. Jankovskaja, K. Wannerberger, T. Arnebrant, Sci. Rep. 2017, 7, 1.

[33] P. J. Flory, J. Rehner, J. Chem. Phys. 1943, 11, 521.

[34] T. Yang, Mechanical and Swelling properties of Hydrogels, 2012.

[35] N. A. Peppas, E. W. Merrill, J. Appl. Polym. Sci. 1977, 21, 1763.

[36] N. A. Peppas, P. Bures, W. Leobandung, H. Ichikawa, Eur. J. Pharm. Biopharm. 2000, 50, 27. 
[37] C. A. Durst, M. P. Cuchiara, E. G. Mansfield, J. L. West, K. J. Grande-Allen, Acta Biomater. 2011, 7, 2467.

[38] M. A. Al-Ameen, G. Ghosh, Biosens. Bioelectron. 2013, 49, 105.

[39] C. A. Deforest, K. S. Anseth, Annu. Rev. Chem. Biomol. Eng. 2012, 3, 421.

[40] G. M. Cruise, D. S. Scharp, J. A. Hubbell, Biomaterials 1998, 19, 1287.

[41] S. Lee, X. Tong, F. Yang, Acta Biomater. 2014, 10, 4167.

[42] E. G. Reynaud, U. Kržič, K. Greger, E. H. K. Stelzer, HFSP J. 2008, 2, 266.

[43] O. E. Olarte, J. Andilla, E. J. Gualda, P. Loza-Alvarez, Adv. Opt. Photonics 2018, $10,111$.

[44] R. Moll, D. L. Schiller, W. W. Franke, J. Cell Biol. 1990, 111, 567.

[45] T. Valenta, B. Degirmenci, A. E. Moor, P. Herr, D. Zimmerli, M. B. Moor, G. Hausmann, C. Cantù, M. Aguet, K. Basler, Cell Rep. 2016, 15, 911.

[46] H. Gehart, H. Clevers, Nat. Rev. Gastroenterol. Hepatol. 2019, 16, 19.

[47] C. A. Thorne, I. W. Chen, L. E. Sanman, M. H. Cobb, L. F. Wu, S. J. Altschuler, Dev. Cell 2018, 44, 624.

[48] G. Altay, E. Larrañaga, S. Tosi, F. M. Barriga, E. Batlle, V. Fernández-Majada, E. Martínez, Sci. Rep. 2019, 9, 1.

[49] D. Serra, U. Mayr, A. Boni, I. Lukonin, M. Rempfler, L. Challet Meylan, M. B. Stadler, P. Strnad, P. Papasaikas, D. Vischi, A. Waldt, G. Roma, P. Liberali, Nature 2019.

[50] Z. Kabiri, G. Greicius, B. Madan, S. Biechele, Z. Zhong, H. Zaribafzadeh, Edison, J. Aliyev, Y. Wu, R. Bunte, B. O. Williams, J. Rossant, D. M. Virshup, Dev. 2014, $141,2206$.

[51] M. Nikolaev, O. Mitrofanova, N. Broguiere, S. Geraldo, D. Dutta, Y. Tabata, B. Elci, N. Gjorevski, H. Clevers, M. P. Lutolf, Nature 2020, in Press.

[52] T. Canal, N. A. Peppas, J. Biomed. Mater. Res. 1989, 23, 1183.

[53] A. Laskin, V. Laskin, Laser Beam Shap. XIV 2013, 8843, 88430C.

[54] C. A. Schneider, W. S. Rasband, K. W. Eliceiri, Nat. Methods 2012, 9, 671.

[55] L. M. Weber, C. G. Lopez, K. S. Anseth, J. Biomed. Mater. Res. A 2009, 90, 720.

[56] F. M. Barriga, E. Montagni, M. Mana, M. Mendez-Lago, X. Hernando-Momblona, M. Sevillano, A. Guillaumet-Adkins, G. Rodriguez-Esteban, S. J. A. Buczacki, M. Gut, H. Heyn, D. J. Winton, O. H. Yilmaz, C. S. O. Attolini, I. Gut, E. Batlle, Cell Stem Cell 2016, 20, 801.

[57] V. Fernández-Majada, P.-S. Welz, M. A. Ermolaeva, M. Schell, A. Adam, F. 
Dietlein, D. Komander, R. Büttner, R. K. Thomas, B. Schumacher, M. Pasparakis, Nat. Commun. 2016, 7, 12508.

[58] H. Khalil, W. Nie, R. A. Edwards, J. Yoo, J. Vis. Exp. 2013, 1.

[59] V. Hagel, T. Haraszti, H. Boehm, Biointerphases 2013, 8, 1.

[60] D. Melekaslan, F. Kasapoglu, K. Ito, Y. Yagci, O. Okay, Polym. Int. 2004, 53, 237.

[61] E. W. Merrill, K. A. Dennison, C. Sung, Biomaterials 1993, 14, 1117.

[62] A. Engler, L. Bacakova, C. Newman, A. Hategan, M. Griffin, D. Discher, Biophys. J. 2004, 86, 617 .

[63] M. Ahearne, Y. Yang, A. J. El Haj, K. Y. Then, K. K. Liu, J. R. Soc. Interface $\mathbf{2 0 0 5}, 2,455$.

\section{Table of contents (ToC)}

Gizem Altay, Aina Abad-Lázaro, Emilio J. Gualda, Jordi Folch, Claudia Insa, Sébastien Tosi, Xavier Hernando-Momblona, Eduard Batlle, Pablo Loza-Álvarez, Vanesa Fernández-Majada, and Elena Martinez*

Modelling biochemical gradients in vitro to control cell compartmentalization in a microengineered 3D model of the intestinal epithelium

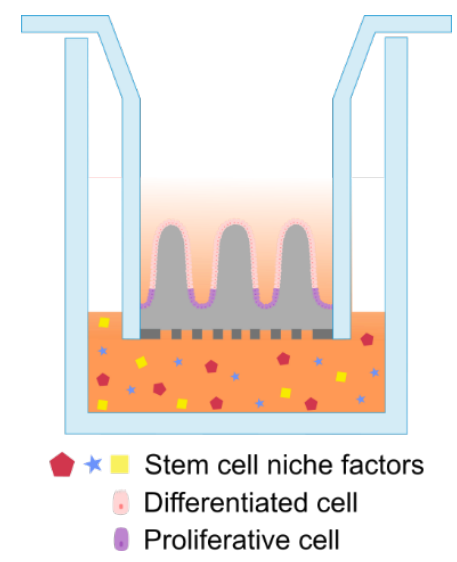

Biomolecular gradients are fundamental in intestinal epithelial tissue organization and function. The microengineered platform developed, with well-controlled spatiotemporal gradients of intestinal stem cell niche factors, 3D architecture and organoid-derived cells retrieves an in vivo-like cell compartmentalization. The system is easily incorporated in 
standard cell culture inserts where one can systematically test factors critical for intestinal tissue dynamics. 